\title{
Consumo alimentar de gestantes adolescentes atendidas em serviço de assistência pré-natal ${ }^{1}$
}

\section{Food consumption of pregnant adolescents assited by prenatal service}

Daniela Vasconcelos de AZEVEDO2

Helena Alves de Carvalho SAM PAIO²

RE S U M O

0 presente estudo analisou o consumo alimentar de 99 gestantes adolescentes atendidas no serviço de assistência pré-natal da Maternidade Escola Assis Chateaubriand, na cidade de Fortaleza, Brasil. Os dados foram levantados por meio de entrevistas no próprio serviço e em visitas domiciliares, com a utilização de um instrumento para obtenção de dados socioeconômicos e do inquérito alimentar recordatório de 24 horas para obtenção de dados alimentares. Os resultados mostraram que as dietas estavam desbalanceadas e monótonas: $63,6 \%$ delas tinham menos de $90,0 \%$ das necessidades energéticas de gestantes e mais da metade estava adequada em seu conteúdo protéico, com excesso de lipídeos e inadequado conteúdo de carboidratos. Alguns micronutrientes, como ferro, cálcio, ácido fólico, zinco e vitamina $\mathrm{B}_{1}$, foram consumidos em quantidades bem abaixo do recomendado. Sugere-se a implementação de ações educativas na área de alimentação e nutrição durante o pré-natal, no sentido de explorar os conhecimentos e práticas alimentares já existentes, auxiliando na escolha de alimentos saudáveis e compatíveis com a situação de vida das gestantes.

Termos de indexação: alimentação, gestação, adolescência.

\section{A B S T R A C T}

This study analyzed the food consumption of 99 pregnant adolescents assisted by Assis Chateaubriand Maternity Hospital, located in the city of Fortaleza, state of Ceará, Brazil. The data were collected by interview

\footnotetext{
${ }^{1}$ Artigo elaborado a partir da dissertação de mestrado em Saúde Pública de D.V. AZEVEDO “Adolescência: gestação, nutrição, conhecimentos, atitudes e práticas associadas", Universidade Estadual do Ceará, 1998. 179p.

${ }^{2}$ Curso de Nutrição, Universidade Estadual do Ceará. R. Paranjana, 1700, Campus do Itapiri, 60740-400, Fortaleza, CE, Brasil. Correspondência para/Correspondence to: D.V. AZEVEDO.E-mail: dami.40@globo.com
} 
at the hospital prenatal service and by home visit. The questionnaire contained questions about socieconomic information and included a 24-hour food recall. Results showed unbalanced and monotonous diets. It was demonstrated that $63.6 \%$ of the diets were low in calories and more than half of them were normal in proteins, high in lipids and low in carbohydrates. Some micronutrients, such as iron, calcium, folic acid, zinc and vitamin $B_{1}$, were lower than the recommended values. The authors suggest the implementation of an education program addressed to the prenatal care service, involving food and nutrition during pregnancy. This program should explore the knowledge and feeding practices that already exist and help the pregnant adolescents to make healthier food choices according to their social level.

Index terms: feeding, pregnancy, adolescence.

\section{N T R O D U Ç Ã O}

O acompanhamento nutricional da mulher durante a assistência pré-natal tem como principais objetivos estabelecer o estado nutricional, identificar fatores de risco, possibilitar interferências terapêuticas e profiláticas no sentido de corrigir distorções e planejar a educação nutricional ${ }^{1}$.

A avaliação nutricional individualizada no início do pré-natal é importante para estabelecer as necessidades de nutrientes neste período e deve ser realizada continuamente ao longo da gravidez². Dentro deste procedimento, a avaliação do consumo alimentar ajuda na detecção de ingestão inadequada e hábitos desfavoráveis 3 .

Durante a gestação, há necessidade adicional de energia por causa do crescimento do feto, da placenta, dos tecidos maternos, bem como para o próprio consumo da gestante ${ }^{1}$. Essas necessidades adicionais de energia na gravidez a termo, em uma mulher eutrófica, com ganho ponderal em torno de $12,5 \mathrm{~kg}$ e bebê com peso ao nascimento superior a $3,0 \mathrm{~kg}$, são estimadas em 80 mil kcal totais ou $300 \mathrm{kcal} / \mathrm{dia}^{4}$. Mesmo assim, é difícil se estabelecer precisamente as necessidades de energia, devido a diversos fatores que estão influenciando o período gestacional, como o peso pré-gravídico, a quantidade e composição do ganho de peso, o estágio da gravidez e o nível de atividade física ${ }^{5}$.

A restrição de energia nesse período pode ocasionar conseqüências negativas ao concepto, pois o crescimento fetal ótimo somente ocorre quando a gestante é capaz de acumular reservas corporais extras 5 .

Com relação à gravidez em adolescentes, não havia até bem pouco tempo recomendações de nutrientes específicos, fazendo com que se utilizassem os mesmos acréscimos de nutrientes recomendados para a gestante adulta na análise da dieta deste grupo 4 .

Nos últimos anos, diversos estudos foram realizados no sentido de determinar novas recomendações de nutrientes para as diversas faixas etárias, incluindo-se a gestante menor de 18 anos. Atualmente já existe a Dietary Reference Intake (DRI) ${ }^{6}$ que incorpora o conceito da antiga Recommended Dietary Allowances (RDA $)^{7} \mathrm{e}$ ainda inclui três outros valores de referência. Nela se encontra atualização dos valores de recomendação para muitos micronutrientes. Recentemente foram publicadas também novas recomendações para energia e macronutrientes, englobando a gestante adolescente ${ }^{8}$.

O tema alimentação de gestante adolescente tem sido pouco explorado. Poucas pesquisas sobre este assunto se encontram disponíveis na literatura e a maioria foi realizada nos Estados Unidos, como o estudo de Stevens-Simon \& M cAnarney (1992) ${ }^{9}$, realizado com 141 gestantes adolescentes negras e de baixa renda, abordando hábitos sociais, dietéticos e de saúde, e o de Skinner et al. (1992) $)^{10}$ sobre dados sociodemográficos e dietéticos de 115 gestantes adolescentes brancas, no último trimestre. Já Dunn et al. $(1994)^{11}$, além de investigarem aspectos semelhantes aos estudos citados, também 
avaliaram preferências e aversões alimentares. No Brasil, não há pesquisas que abordem o tema alimentação e nutrição de adolescentes durante a gestação.

Diante do exposto, procurou-se investigar e analisar o consumo alimentar de gestantes adolescentes atendidas em um serviço público de referência para assistência pré-natal em Fortaleza, Ceará, Brasil.

\section{CASUÍSTICA M MÉTODOS}

A M aternidade Escola Assis Chateaubriand (M EAC), localizada na cidade de Fortaleza, Ceará, atende no seu Serviço de Tocoginecologia Infanto-Puberal adolescentes até 19 anos provenientes da capital e também do interior do Estado. A equipe de profissionais que atende na assistência pré-natal é composta de ginecologistas-obstetras, enfermeiro, psicólogo, assistente social, dentista e pediatra. 0 atendimento é realizado duas vezes por semana, nos dois turnos, sendo agendadas 40 consultas por turno (20 para cada ginecologista-obstetra). Em cada dia de atendimento são destinadas 20 vagas (correspondendo $25 \%$ das consultas diárias) à gestantes que estão procurando - Serviço pela primeira vez, para iniciar o acompanhamento pré-natal, independentemente da semana gestacional em que se encontrem.

A casuística compreendeu 99 gestantes adolescentes pertencentes à demanda espontânea, as quais estavam realizando a primeira consulta de pré-natal, no período de outubro de 1997 a março de 1998. Optou-se por selecionar gestantes em primeira consulta a fim de evitar identificar hábitos alimentares que já houvessem sofrido influências de orientações recebidas no Serviço.

Os dados foram levantados pelos próprios pesquisadores, por meio de entrevistas no Serviço, antes das consultas, e posteriormente em visitas domiciliares. As gestantes responderam a perguntas estruturadas relacionadas à situação socioeconômica (renda familiar, escolaridade e condições de moradia) e dados de ingestão alimentar foram obtidos por meio do método de inquérito alimentar recordatório de 24 horas, realizado em três diasnão consecutivos, incluindo-se um dia de final de semana. O primeiro recordatório foi realizado no Serviço, antes da consulta de pré-natal, e os outros dois na residência da gestante. $A$ análise foi efetuada utilizando-se a média dos três recordatórios. Os alimentos consumidos foram relatados pelas gestantes em medidas caseiras, as quais foram posteriormente transformadas em peso (gramas), utilizando-se as padronizações da tabela de Pinheiro et al. (1994) ${ }^{12}$. Os alimentos citados que não constavam na referida tabela foram adquiridos em supermercados locais e pesados em balança da marca Bender ${ }^{\circledR}$ com capacidade para $10 \mathrm{~kg}$.

Identificou-se a idade gestacional no início do pré-natal e foram aferidos o peso e a altura corporais. Considerou-se como primeiro trimestre gestacional até a $12^{\text {a }}$ semana, segundo trimestre da $13^{a}$ até a $28^{a}$ e terceiro trimestre da 29ạ até a 42 a semana gestacional ${ }^{13}$. Para avaliar o estado nutricional, utilizou-se o Índice de M assa Corporal (IMC) anterior à gestação com o peso pré-gravídico estimado, conforme recomendação de Diniz $(1994)^{11}$, adotando-se o seguinte critério de classificação: baixo peso - IMC $<19,8$; peso adequado - IMC = 19,8 - 26; sobrepeso - IMC>26 - 29 e obesidade - IM C >29, segundo Gutierrez \& King $(1994)^{4}$.

O critério de adequação do consumo alimentar adotado para energia e proteínas foi de $2500 \mathrm{kcal} / \mathrm{dia}$ e $60 \mathrm{~g} / \mathrm{dia}$, respectivamente, padrão adotado inclusive para as três gestantes menores de 15 anos $^{4}$. A composição química das dietas foi analisada em relação a vitaminas (A, $C, B_{1}, B_{2}$, ácido fólico e $B_{12}$ ) e minerais (cálcio, ferro e zinco), usando como parâmetro a Dietary Reference Intake (DRI) ${ }^{6}$. Optou-se por não utilizar a nova DRI como parâmetro para energia e proteínas, pois sua definição é muito recente e não seria possível o confronto dos dados obtidos com outros relatos da literatura. Foi adotado o tema "adequação" para expressar o percentual de atendimento aos valores de referência citados. 
Para micronutrientes, foram utilizadas as orientações de Earl \& Borra $(2000)^{14}$, segundo os quais os indivíduos devem atingir 100\% de adequação. Como havia dietas com adequação bem abaixo do recomendado, resolveu-se subdividir as faixas em: $<70 \%$ (muito inadequada), $70 \%-<100 \%$ (inadequada) e $\geq 100 \%$ (adequada).

A definição de normalidade de energia e macronutrientes seguiu os critérios da World Health Organization (1990) ${ }^{15}$, que estabelece ingestão de energia em relação às necessidades diárias $<90 \%$ como insuficiente, entre $90 \%-110 \%$ como adequada e $>110 \%$ como excessiva. De acordo com os referidos critérios, considerou-se a ingestão de hidratos de carbono em relação ao total energético $<55 \%$ como insuficiente, entre $55 \%-75 \%$ como adequada e $>75 \%$ como excessiva; determinando-se o consumo de proteínas em relação ao total energético $<10 \%$ como insuficiente, entre $10 \%$ - 15\% como adequada e $>15 \%$ como excessivo; e identificou-se o consumo de lipídeos em relação ao total energético $<15 \%$ como insuficiente, entre $15-30 \%$ como adequado e $>30 \%$ como excessivo.

Além de analisar a dieta de acordo com energia e nutrientes, foram observados também a composição básica da dieta e o número de refeições realizadas pelas gestantes, por meio de análise qualitativa do consumo.

Para auxiliar na avaliação da composição química das dietas, empregou-se o Programa Sistema de Apoio à Decisão em Nutrição (versão 2.5) do Centro de Informática em Saúde da Escola Paulista de Medicina. Somente para alguns alimentos que não constavam neste programa foram consultadas as tabelas de composição de alimentos de Franco (1989) ${ }^{16}$ e Pinheiro et al $(1994)^{12}$, ou os rótulos dos referidos produtos. $\mathrm{Na}$ análise dos dados gerais utilizou-se o programa Epi Info versão 6.0.

\section{RESULTADOSE DISCUSSÃ O}

As gestantes entrevistadas tinham em média 16,4 anos, variando de 14 a 19 anos, e eram provenientes da periferia da capital - Fortaleza ou de municípios circunvizinhos. Suas residências eram modestas, constituídas por um ou dois cômodos. Grande parte destas moradias $(86,5 \%)$ possuía água proveniente do abastecimento público, embora nem sempre dentro de seu domicílio. Cerca da metade das residências $(49,0 \%$ ) possuía esgoto sanitário ligado à rede pública. A maior concentração da renda total das famílias estava na faixa de um a três salários mínimos (51,5\%). Apenas $11,1 \%$ das gestantes conseguiram chegar ao ensino médio e $63,6 \%$ não estavam estudando no momento da pesquisa.

Em se tratando do início do pré-natal, somente $20,2 \%$ das gestantes o fizeram no primeiro trimestre e a maioria $(71,7 \%)$, no segundo trimestre. 0 restante, $8,1 \%$, iniciou 0 acompanhamento no terceiro trimestre.

A análise do IMC mostrou que, embora mais da metade das adolescentes $(69,7 \%)$ tenha iniciado a gravidez com peso adequado para a altura, 25,2\% delas iniciaram com baixo peso. Apenas $3,1 \%$ das gestantes apresentavam sobrepeso e $2,0 \%$, obesidade.

De acordo com a avaliação da adequação energética, 63,6\% das dietas tinham menos de $90 \%$ das necessidades energéticas, $22,2 \%$ tinham entre $90,0 \%$ e $110,0 \%$ e as demais, $14,1 \%$, tinham mais de $110,0 \%$ destas necessidades. $A$ média de ingestão energética das adolescentes foi de $2347 \mathrm{kcal}$. Carruth \& Skinner $(1991)^{7}$ encontraram média de consumo energético de $2249 \mathrm{kcal}$. Outros autores também observaram em seus estudos com gestantes adolescentes, ingestão energética abaixo do recomendado 9,18-20. Ramirez et al. (1998) ${ }^{21}$ detectaram um elevado percentual $(70,0 \%)$ de gestantes adolescentes com consumo adequado em energia.

A maioria das dietas revelou-se com conteúdo protéico adequado, excessivo em lipídeos e inadequado em carboidratos (Tabela 1). A proporção de proteínas, em relação ao valor energético, apresentou-se adequada, embora 0 valor médio de consumo estivesse acima do 
recomendado (Tabela 2). 0 consumo elevado de proteínas foi detectado em outras pesquisas realizadas com gestantes ${ }^{9-11,17}$. No estudo de Carruth \& Skinner (1991) ${ }^{17}$, a média de consumo de proteína excedeu o recomendado, atingindo $146,6 \%$. Ramirez et al. (1998) ${ }^{21}$ encontraram $70 \%$ das gestantes de seu estudo com consumo adequado em proteínas. Contrariamente aos estudos citados, Chaturvedi et al. (1994) ${ }^{19}$ encontraram consumo deficiente de proteínas em $25 \%$ a $37 \%$ das dietas de gestantes adolescentes casadas, da zona rural da Índia.

Tabela 1. Distribuição energética dos macronutrientes na dieta das gestantes pesquisadas. Fortaleza, 1998.

\begin{tabular}{lcr}
\hline Faixa de distribuição (\%) & $\mathrm{n}$ & $\%$ \\
\hline Proteínas & & \\
$<10$ (inadequado) & 8 & 8,1 \\
$10-15$ (adequado) & 23 & 68,7 \\
$>15$ (excessivo) & 99 & 23,2 \\
\hline Total & & 100,0 \\
\hline Lipídeos & 1 & \\
$<15$ (inadequado) & 32 & 32,0 \\
$15-\quad 30$ (adequado) & 66 & 66,7 \\
$>30$ (excessivo) & 99 & 100,0 \\
\hline Total & & \\
\hline Hidratos de carbono & 58 & 58,6 \\
$<55$ (inadequado) & 40 & 40,4 \\
$55 \quad$ 75 (adequado) & 1 & 1,0 \\
$>75$ (excessivo) & 99 & 100,0 \\
\hline Total & & \\
\hline
\end{tabular}

$(\mathrm{n}=99)$.
O excesso de lipídeos pode ser justificado pelo consumo de frituras nas preparações de carnes em geral (incluindo frango e peixes). Skinner et al. (1992) encontraram resultados semelhantes ao do presente trabalho, com proporções de carboidratos e lipídeos da dieta de adolescentes grávidas, $50 \%$ e $36 \%$, respectivamente, em relação ao valor energético total.

A análise da adequação de micronutrientes, indicou que as dietas estavam muito inadequadas ( $<70 \%$ ) em relação ao cálcio, ferro, zinco, vitamina $\mathrm{B}_{1}$ e ácido fólico (Figura 1 ). Somente a vitamina $\mathrm{C}$ e a vitamina $\mathrm{A}$ apresentaram resultados melhores, com 53,3\% e 58,2\% das dietas, respectivamente, atingindo a faixa considerada adequada ( $\left.{ }^{3} 100 \%\right)$.

A deficiência da maioria dos micronutrientes das dietas analisadas pode perfeitamente ser explicada pelo baixo consumo de frutas, hortaliças, leite e seus derivados. Embora o consumo de frutas tenha sido baixo, as mais consumidas foram manga e acerola, o que pode explicar as vitaminas $\mathrm{C}$ e A acima das recomendações. Tais resultados vão ao encontro dos obtidos em pesquisas semelhantes, as quais também detectaram deficiência de ferro 10,11,17,20-22, zinco 20,23, ácido fólico ${ }^{10,11,20}$ e cálcio ${ }^{11,17,20,21,23,24}$. A vitamina C também estava acima das recomendações em alguns desses estudos ${ }^{10,11,17}$.

Tabela 2. Consumo médio de energia e nutrientes das gestantes entrevistadas, em relação aos valores de referência ${ }^{1}$. Fortaleza, 2002.

\begin{tabular}{lcr}
\hline Energia / nutrientes & Necessidades nutricionais & Consumo médio / variação \\
\hline Energia $(\mathrm{kcal})$ & 2500 & $2347,0(800-5553)$ \\
Proteínas $(\mathrm{g})$ & 60 & $75,0(21,9-240,0)$ \\
Cálcio $(\mathrm{mg})$ & 1300 & $586,6(129,7-1369,0)$ \\
Ferro $(\mathrm{mg})$ & 27 & $12,3(4,3-34,1)$ \\
Zinco $(\mathrm{mg})$ & 13 & $8,8(0,9-26,7)$ \\
Vitamina C $(\mathrm{mg})$ & 80 & $219,7(1,6-2236,1)$ \\
Vitamina $\mathrm{B}_{1}(\mathrm{mg})$ & 1,4 & $1,0(0,4-2,4)$ \\
Vitamina $\mathrm{B}_{2}(\mathrm{mg})$ & 1,4 & $1,3(0,4-4,9)$ \\
Ácido fólico $(\mu \mathrm{g})$ & 400 & $96,5(11,2-391,1)$ \\
Vitamina $\mathrm{B}_{12}(\mu \mathrm{g})$ & 2,2 & $2,6(0,2-7,9)$ \\
Vitamina A $(\mathrm{RE})$ & 800 & $820,8(57,5-4006,9)$ \\
\hline
\end{tabular}

${ }^{1}$ RDA $(1989)^{17}$ para energia e proteínas e DRI $(2002)^{8}$ para demais nutrientes. 


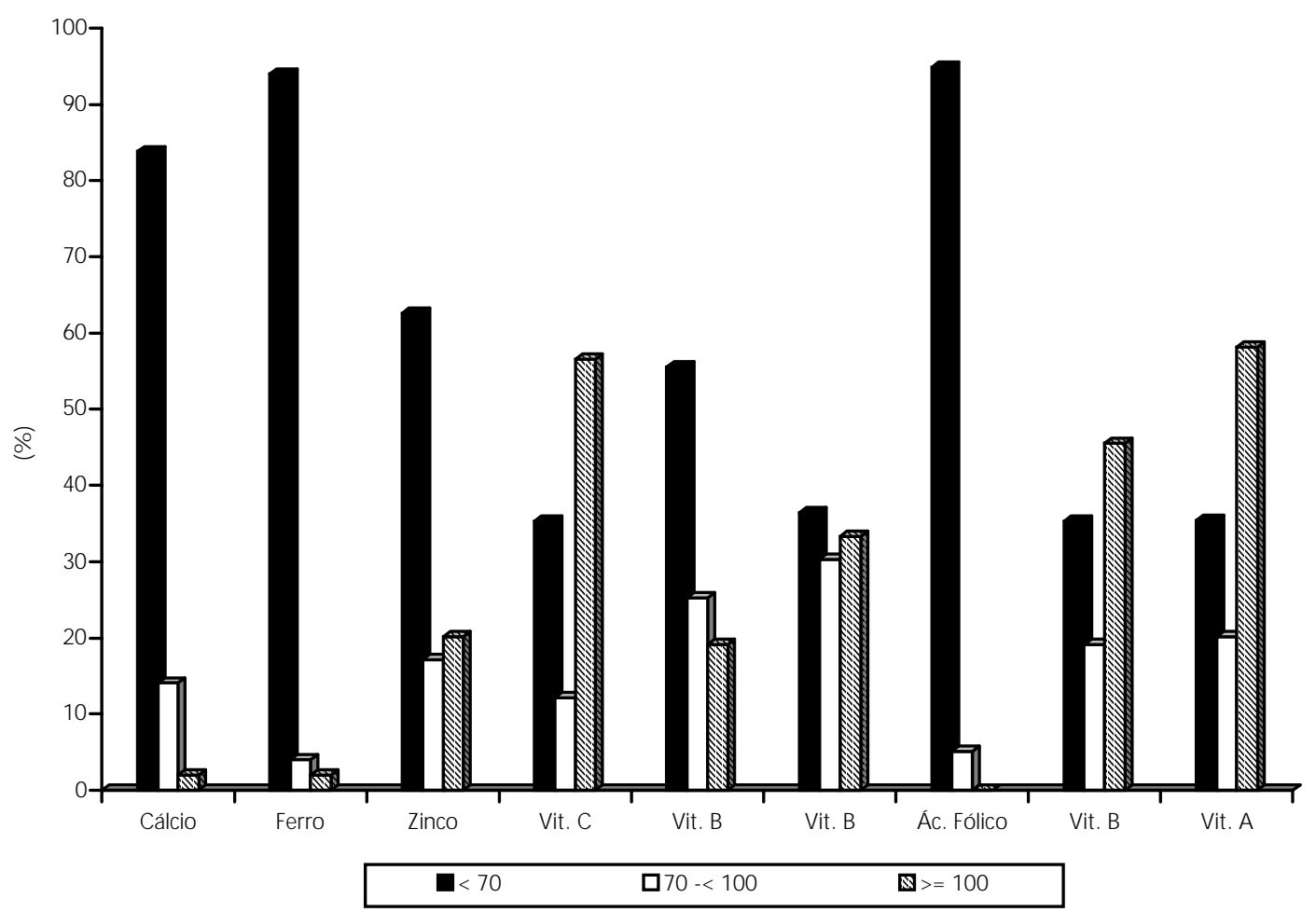

Figura 1. Adequação dos micronutrientes das dietas das gestantes, segundo os valores de referênciaª . Fortaleza, 2002.

(1) DRI $(2002)^{8}$.

Nota: Vit = Vitamina; $\mathrm{ac}=$ ácido.

De acordo com a média dos três recordatórios de 24 horas, 39,4\% das gestantes realizavam de duas a quatro refeições ao longo do dia e as demais, de cinco a sete. Embora a maioria realizasse o número de refeições recomendado pela literatura ${ }^{1,5}$, a quantidade e a qualidade, como já foi evidenciado, não correspondiam ao considerado adequado para esta faixa etária e para este estado fisiológico.

Observou-se que o desjejum era composto de café e pão com margarina ou cream cracker com margarina, sendo algumas vezes acrescido de leite. No almoço havia sempre arroz, feijão, macarrão e um tipo de carne, sendo a bovina e a de frango as mais consumidas. 0 jantar, quando não repetia o almoço, era composto de pão com margarina e café ou pão com margarina e café com leite. Nos lanches da manhã e tarde havia pão ou bolachas com margarina, acompanhados de café, uma fruta ou um suco.
Constatou-se que $10,1 \%$ das gestantes não consumiram frutas e $63,7 \%$ consumiram quantidades insuficientes (cerca de uma fruta ou um copo de suco por dia). As hortaliças, o leite e seus derivados estavam ausentes em 56,6\% e $14,1 \%$ das dietas das gestantes, respectivamente. Segundo estudo realizado por Di Cintio et al. $(2001)^{25}$, uma dieta pobre em frutas, vegetais, leite e seus derivados, e ao mesmo tempo rica em gorduras, pode estar relacionada ao aumento do risco de abortos espontâneos ou mesmo ser fator determinante desse risco.

Verificou-se pouca variação no cardápio diário, ocorrendo situações nas quais todos os três recordatórios foram praticamente iguais tanto no tipo quanto na quantidade dos alimentos consumidos, tornando assim as dietas monótonas. Em princípio, pode-se esperar que essas gestantes tenham uma alimentação inadequada tanto em quantidade quanto em qualidade, se for levado 
em conta apenas a questão da renda familiar. No entanto, apesar da forte e indiscutível influência da renda, hábitos alimentares, conhecimentos sobre alimentação e nutrição também interferem na escolha e composição da alimentação diária, principalmente em uma fase tão delicada como é a gestação.

Diante das discussões, conclui-se que as gestantes avaliadas não estão consumindo uma dieta balanceada, indicando a necessidade de ações educativas com abordagem da situação socioeconômica e dos hábitos alimentares, de forma a auxiliá-las na seleção de alimentos compatíveis com seu estado fisiológico.

\section{REFERÊ N CIAS}

1. Diniz LEV. Nutrição e gravidez. In: Zugaib BM, Sancrovski M. O pré-natal. Rio de Janeiro: Atheneu; 1994. p.71-6.

2. Williams SR. Orientação nutricional na assistência pré-natal. In: Worthington-Roberts BS, Vemeerchi J, Williams SR. Nutrição na gravidez e lactação. Rio de Janeiro: Interamericana; 1986. p.105-35.

3. Mikode MS, White AA. Dietary assessment of middle-income pregnant women during the first, second, and third trimesters. J Am Diet Assoc 1994; 94(2):196-99.

4. Gutierrez $Y$, King JC. Nutrition during teenage pregnancy. Pediatr Ann 1993; 22(2):99-108.

5. Neuhouser MLS. Nutrition during pregnancy and lactation. In: Mahan LK, Escott-Stump S. Krause's food, nutrition, and diet therapy. Philadelphia: W.B. Saunders; 1996. p.181-201.

6. Dietary Reference Intake: Application in Dietary Assessment. Institute of Medicine (IOM) [online] 2001 [cited 20-9-2001]. Avaliable from: http// www.books.nap.edu/books/0309071836/html/ 287.html\#pagetop

7. Dietary Reference Intake for energy, carbhoidrates, fiber, fat, protein and amino acids (M acronutrients). Institute of Medicine (IOM) [online] 2002 [cited 5-10-2002]. Avaliable from: http://w w w.nap.edu/openbook/0309085373/ $\mathrm{html} / 1 . \mathrm{htm}$

8. Monsen ER. The 10th edition of the RDA: What's new in the 1989 RDA? J Am Diet Assoc 1989; 89:1748-52.

9. Stevens-Simon C, M CAnarney ER. Determinants of weight gain in pregnant adolescents. J Am Diet Assoc 1992; 92(11):1348-51.

10. Skinner JD, Carruth BR, Pope J, Varner L, Goldberg D. Food and nutrient intake of white, pregnant adolescents. J Am Diet Assoc 1992; 92(9): 1127-9.

11. Dunn C, Kolasa K, Dunn PC, Ogle M B. Dietary intake of pregnant adolescents in a rural southern community. J Am Diet Assoc 1994; 94(9): 1040-1.

12. Pinheiro ABV, Lacerda EMA, Benzecry EH, Gomes MCS, Costa VM . Tabela para avaliação de consumo alimentar em medidas caseiras. Rio de Janeiro; 1994.

13. M artins-Costa S, Ramos JG, Chaves EM . Assistência ao pré-natal normal e de alto risco. In: Freitas, F, Martins-Costa S, Ramos JGL, Passos EP. Rotinas em obstetrícia. Porto Alegre: Artes M édicas; 1993. p.1-12.

14. Earl R, Borra ST. Guidelines for dietary planning. In: Mahan LK, Escott-Stump S. Krause's food, nutrition, and diet therapy. Philadelphia: W.B. Saunders; 2000. p.332-52.

15. World Health Organization. Diet, nutrition and the prevention of chronic diseases. Geneva; 1990. (Technical Report Series 797).

16. Franco G. Tabela de composição dos alimentos. Rio de Janeiro: Atheneu; 1989.

17. Carruth BR, Skinner JD. Pregnant adolescents report infrequent use of sugar substitutes. J Am Diet Assoc 1991; 91(5):608-10.

18. Boggio JF, Quiroz J, Calderón EF. Estado nutricional de la gestante adolescente y del recién nacido y consumo de alimentos y nutrientes. Ginecol Obstet 1997; 43(1):9-15.

19. Chaturvedi S, Kapil U, Bhanthi T, Gananasekaran $\mathrm{N}$, Pandey RM. Nutritional status of married 
adolescent girls in rural Rajasthan. Indian J Pediatr 1994; 61(6):695-701.

20. Giddens JB, Krug SK, Tsang RC, Guo S, Miodovnik M, Pradda JA. Pregnant adolescent and adult women have similarly low intakes of selected nutrients. J Am Diet Assoc 2000; 100(11): 1334-40.

21. Ramirez JP, Díaz J, Inga M, Muñoz C. Perfil alimentario-nutricional de la gestante adolescente. Ginecol Obstet 1998; 44(1):40-4.

22. Hertrampf ED, Olivares MG, Letelier AC, Castillo DC. Iron nutritional status in pregnant adolescents at the beginning of gestation. Rev M ed Chil 1994; 122(12):1372-7.
23. Borghi R, Baxter IC, Galleta M AK, M arculeviciuis J, Zugaib M. Mudanças no comportamento alimentar em gestantes adolescentes. Rev Ginecol Obstet 1997; 8(1):20-2.

24. Perez MSS, Sanchez SLA. Adolescent pregnancy a proposal for intervention. Rev Enfer 1997; 20(229):10-5.

25. Di Cintio et al. Dietary factors and risk of spontaneous abortion. Eur J Gynecol Reprod Biol 2001; 95(1):132-6.

Recebido em 3 de setembro de 2001 e aceito em 23 de junho de 2003. 